\title{
Widespread, focal copy number variations (CNV) and whole chromosome aneuploidies in Trypanosoma cruzi strains revealed by array comparative genomic hybridization
}

\author{
Todd A Minning ${ }^{1}$, D Brent Weatherly' ${ }^{1}$ Stephane Flibotte², Rick L Tarleton ${ }^{1 *}$
}

\begin{abstract}
Background: Trypanosoma cruzi is a protozoan parasite and the etiologic agent of Chagas disease, an important public health problem in Latin America. T. cruzi is diploid, almost exclusively asexual, and displays an extraordinarily diverse population structure both genetically and phenotypically. Yet, to date the genotypic diversity of $T$. cruzi and its relationship, if any, to biological diversity have not been studied at the whole genome level.

Results: In this study, we used whole genome oligonucleotide tiling arrays to compare gene content in biologically disparate $T$. cruzi strains by comparative genomic hybridization (CGH). We observed that $T$. cruzi strains display widespread and focal copy number variations (CNV) and a substantially greater level of diversity than can be adequately defined by the current genetic typing methods. As expected, CNV were particularly frequent in gene family-rich regions containing mucins and trans-sialidases but were also evident in core genes. Gene groups that showed little variation in copy numbers among the strains tested included those encoding protein kinases and ribosomal proteins, suggesting these loci were less permissive to CNV. Moreover, frequent variation in chromosome copy numbers were observed, and chromosome-specific CNV signatures were shared by genetically divergent T. cruzi strains.

Conclusions: The large number of CNV, over 4,000, reported here uphold at a whole genome level the long held paradigm of extraordinary genome plasticity among T. cruzi strains. Moreover, the fact that these heritable markers do not parse T. cruzi strains along the same lines as traditional typing methods is strongly suggestive of genetic exchange playing a major role in T. cruzi population structure and biology.
\end{abstract}

\section{Background}

Human infection with $T$. cruzi, a vector-borne protozoan parasite, is the cause of Chagas disease, which is a potentially fatal malady endemic to much of Latin America. T. cruzi is a member of the order Trypanosomatida, and, like other members of this early diverged group of eukaryotes, is diploid and has a primarily clonal population structure which is extremely diverse [1]. The broad host range of T. cruzi, which includes over 100 species of both wild and domestic mammals

\footnotetext{
* Correspondence: tarleton@cb.uga.edu

${ }^{1}$ Center for Tropical and Emerging Global Diseases, University of Georgia, Athens, Georgia, 30602, USA

Full list of author information is available at the end of the article
}

(reviewed in [2]) may also contribute to its remarkable phenotypic and genetic diversity.

The population structure of $T$. cruzi has been examined by sub-genomic methods, such as multi-locus sequence typing (MLST) and microsatellite analyses, resulting in the classification of $T$. cruzi strains into six discrete typing units (DTU), type I and types IIa-e (reviewed in [3]). A change in nomenclature from these DTU names to TCI-TCVI has recently been suggested [4]. However, in the interest of clarity we will heretofore use the more familiar nomenclature of type I and types IIa-IIe. Although an asexual replication mechanism best explains the population structure of T. cruzi as it is currently understood, molecular analyses support the occurrence of at least two hybridization events in the

\section{Biomed Central}


past resulting in mosaic genomes in two of the DTU (types IIa and IIc) and hybrid genomes in another two (types IId and IIe), one of which (CL-Brener-type IIe) was used as the type strain for the T. cruzi genome sequencing effort [5].

The genotypic diversity evidenced by sub-genomic methods of analysis suggests that whole genome analyses of $T$. cruzi strain diversity would be fruitful, especially for elucidating the underpinnings of strain diversity in biological characteristics, such as variability in complements of large gene family members. Nearly one half of the $T$. cruzi genome contains repeat sequences largely comprised of thousands of members of large gene families, including trans-sialidases, mucin associated surface proteins (MASP), mucins, retrotransposon hotspot (RHS) proteins, dispersed gene family 1 proteins (DGF), and surface protease gp63 [6]. Many of these have been shown to be important targets of immune responses in infected hosts $[7,8]$. Isolates of $T$. cruzi (generally referred to as "strains") appear to show a near limitless range of variation in important biological characteristics, among these, the numbers of parasites in the blood and tissues of various hosts, the focus and location of inflammation and thus the morbidity and mortality in these hosts, and susceptibility of these isolates to anti-T. cruzi drugs. The current genetic classification cannot fully account for this variation (e.g. all type I strains are not equally virulent) despite the fact that a substantial proportion of this variation is almost certainly based upon genetic differences among isolates.

To date only the reference strain, CL-Brener, has been fully sequenced [6]. Moreover, the genome sequence of the CL-Brener strain has only recently been assembled into chromosome-sized pieces that will facilitate genome-wide strain comparisons [9]. To further explore the degree of genetic variability between $T$. cruzi isolates, and to examine the relationship between genotypic and biological diversity on a genomic scale, we used whole genome oligonucleotide tiling arrays to determine copy number variations (CNV) in 16 T. cruzi strains by competitive hybridizations using the CL-Brener strain as reference. Our results partially support the proposed type I - type II dichotomy in the T. cruzi population structure but also reveal similarities and differences between $T$. cruzi strains that cannot be explained by the current DTU scheme. These findings suggest that either coevolution of distinct, chromosome-specific CNVs frequently occurs in different T. cruzi strains or that chromosome exchange between $T$. cruzi strains is much more common than currently thought. The results also suggest that $T$. cruzi is remarkably permissive to substantial $\mathrm{CNV}$, including whole chromosome CNV.

\section{Results}

Whole genome oligonucleotide tiling arrays $(\sim 290,000$ spots) were designed as described in the Methods section, using the only fully sequenced T. cruzi strain, the hybrid CL Brener, as a template. Because of the hybrid nature of the CL Brener strain, with its "Esmeraldo-like" (Esm) and "non-Esmeraldo-like" (non-Esm) alleles, it was possible to design allele-specific probes for many regions of the genome. Regions of the genome that are rich in genes that are members of large gene families have relatively few probes because the sequence similarities among family members did not permit the design of gene-specific probes.

As a validation of the accuracy of the arrays we performed hybridizations comparing wt $T$. cruzi strains with gene knockout parasite lines generated in our laboratory [10]. Additional file 1 shows the CGH data for one of these hybridizations comparing a knockout strain for enoyl-CoA hydratase/isomerase family protein (tandem genes each singly replaced - [Tc00.1047053511529.160 and Tc00.1047053511529.150]) vs wt T. cruzi. The CNV resulting from single knockout of one copy each of these two tandemly arrayed genes is obvious at the whole chromosome view of chromosome 35, panel A. Moreover, the close-up view in panel $B$ reveals overlap of the Esm and non-Esm probes (green and blue spots, respectively) within the coding portions of the genes, but divergence of Esm and non-Esm ratios for the intergenic region. This reflects the fact that the Esm and non-Esm alleles for these two genes are nearly identical, but the Esm and non-Esm sequences for the intergenic region are not.

Genomic DNA samples from $16 T$. cruzi strains were compared to genomic DNA from the CL-Brener strain by competitive hybridizations on this array and the complete set of figures for all 41 chromosomes for each of the 16 strain comparisons are shown in Additional file 2 and the full dataset can be viewed in Additional files 3 and 4 . The data have also been deposited in the Gene Expression Omnibus (http://www.ncbi. nlm.nih.gov/geo/) under the accession GSE23576. The most striking feature of these results is the very large number of both segmental (ranging in size from 500 bp to over $500 \mathrm{kbp}$ ) and whole chromosome aneuploidies, representative examples of which are shown in the hybridization results for chromosome 39 for 12 strains (Figure 1). Probes with log2ratios approximating zero are present in equal copy numbers in both the test and reference (CL Brener) strains and probes with positive log2ratios have higher copy number in the test strains versus the reference strain, and thus represent amplifications in the test strain. Probes with negative $\log 2$ ratios indicate deletions in the test strain and/or sufficient sequence divergence to result in decreased hybridization in the test channel. Segmental 




aneuploidies (e.g. a $500 \mathrm{kbp}$ segment in the Brazil strain [arrow]) and shared, focal deletions in multiple strains are readily evident (Figure 1 and Additional file 2 ). Sequence divergence resulting in decreased hybridization intensity can be seen in the Esmeraldo panel where the green dots (representing Esmeraldo allelespecific probes) have positive ratios and blue dots (non-Esmeraldo probes) have negative log2ratios (Figure 1). However, note that the average $\log 2$ ratio for all probes is near zero in the Esmeraldo panel, suggesting that Esmeraldo is homozygous for the Esmeraldo haplotype as would be expected and that the total number copies of chromosome 39 in this strain is 2 (assuming, of course, that CL-Brener has 2 copies of chromosome 39). The presence of apparent deletions in the test strains that are shared between many genetically divergent test strains (as seen in the red boxed regions) suggests that such instances are bona fide deletions rather than sequence divergence.
To identify the regions of the $T$. cruzi genome that are most prone to $\mathrm{CNV}$, we annotated segments of the genome that had CNVs relative to the overall average of all strains (Figure 2). 'Hotspots' of CNV were readily evident and were present on every chromosome, although much more prevalent on some (e.g. 18, 38 and 41) than others (e.g. 11, 34, 36, 37, 39). The CNV were also focal and widespread, frequently associated with gene family rich regions of the genome (note that chromosomes 18, 38 , and 41 are the most gene family rich chromosomes), but also in core regions. Due to the scarcity of probes within the gene family rich regions a statistical test of the significance of the bias of hotspot regions being located in gene family rich regions of the genome was not possible.

The classes of genes mapping to these hotspot regions are ranked in order of frequency of CNV in Table 1 (for all gene groups comprised of 5 or more genes, each with a minimum of 5 probes per $500 \mathrm{bp}$ ). Perhaps not 


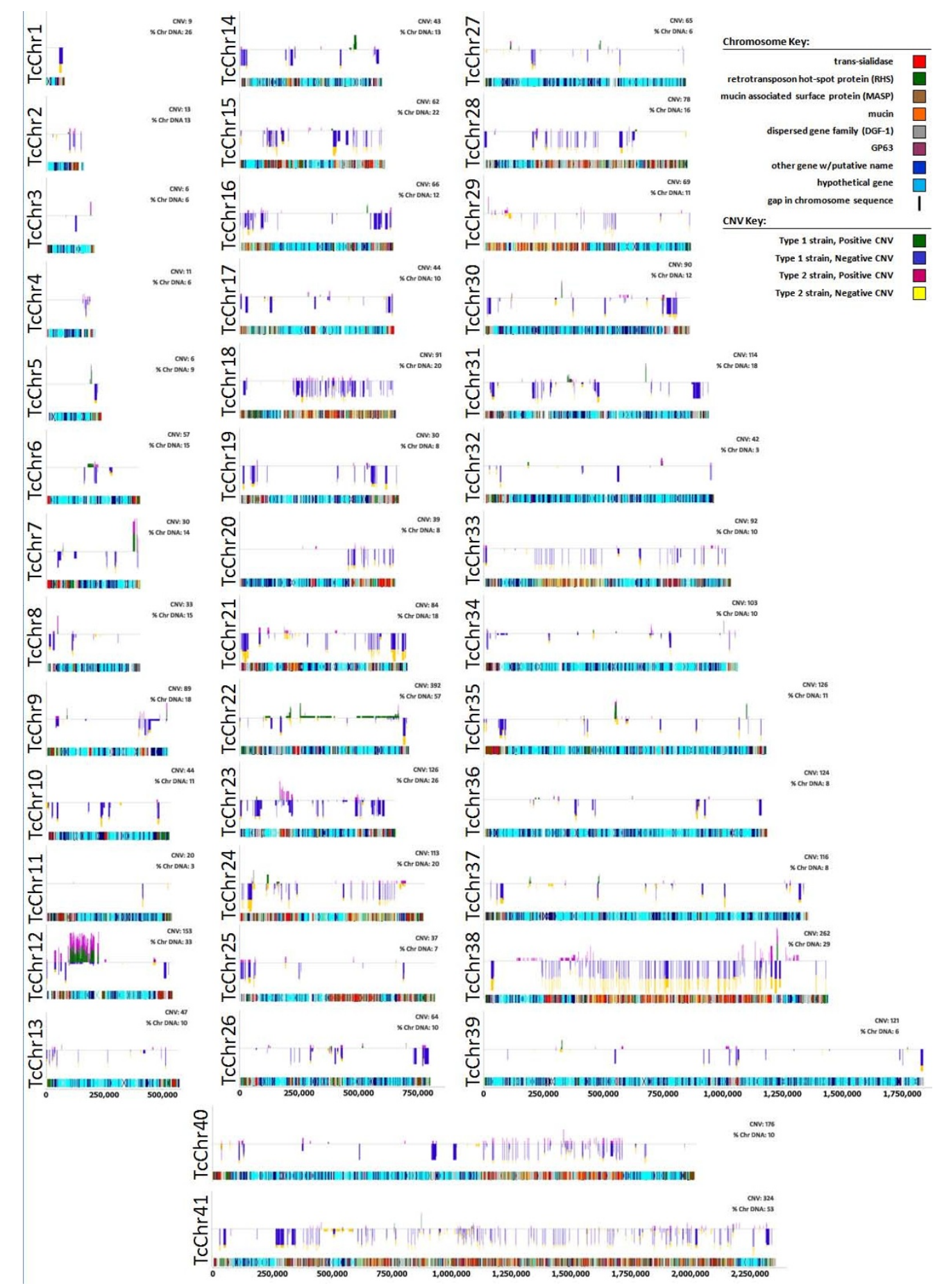

Figure 2 Graphical view of proportion of strains displaying CNV for each sequence feature on each chromosome. A sequence feature is defined as either an annotated gene or the un-translated region (UTR) between two genes. CNV criteria were as follows: minimum log2 ratio of signal intensities (test strain/reference) + /- 0.6, minimum number of probes 5, tolerance for extending a CNV 0.1. At the top of each panel is a line drawing representing a chromosome. Vertical bars represent CNV that met the criteria for significance. Vertical bars above the line are amplifications and those below the line are deletions relative to CL-Brener. The height (depth) of the vertical bars is proportional to the number of strains showing that CNV. The vertical bars are colored to indicate strain type (type 1 and type 2) as follows: green, type 1 strains amplification; blue, type 1 strains deletion; maroon, type 2 strains amplification; yellow, type 2 strains deletion. Below each chromosome line drawing is a diagram representing the annotated chromosome. Genes are color coded based on annotation; named genes not belonging to large gene families (dark blue), hypothetical genes (light blue), trans-sialidases (red), mucin associated surface proteins (MASP) (burnt orange), mucins (orange), retrotransposon hotspot (RHS) proteins (green), dispersed gene family 1 (DGF) (gray), surface protease gp63 (purple). 
Table 1 Distribution of genes associated with hotspot regions, minimum of 5 candidates on the arrays

\begin{tabular}{|c|c|c|c|c|c|}
\hline Gene Name & \# Genome Occurrences $^{1}$ & \#Array Occurrences $^{2}$ & \# Array Candidates ${ }^{3}$ & \# Sig CNV ${ }^{4}$ & $\%$ Sig of Candidates \\
\hline mucin TcMUCII & 728 & 506 & 164 & 121 & 73.7 \\
\hline serine carboxypeptidase & 7 & 7 & 6 & 4 & 66.6 \\
\hline tryptophanyl-tRNA synthetase & 23 & 8 & 6 & 4 & 66.6 \\
\hline mucin-associated surface & 1377 & 948 & 437 & 243 & 55.6 \\
\hline retrotransposon hot & 753 & 419 & 82 & 41 & 50 \\
\hline ATP-dependent chaperone & 5 & 5 & 5 & 2 & 40 \\
\hline beta galactofuranosyl & 66 & 47 & 10 & 4 & 40 \\
\hline mitogen-activated protein kinase & 11 & 11 & 10 & 4 & 40 \\
\hline Sialidase & 3209 & 789 & 135 & 51 & 37.7 \\
\hline clathrin coat & 7 & 7 & 7 & 2 & 28.5 \\
\hline surface protease & 425 & 258 & 88 & 25 & 28.4 \\
\hline myosin heavy & 19 & 19 & 17 & 3 & 17.6 \\
\hline PIF1 helicase-like & 12 & 12 & 12 & 2 & 16.6 \\
\hline mismatch repair & 13 & 13 & 13 & 2 & 15.3 \\
\hline ATP-dependent DEAD/H & 106 & 88 & 66 & 10 & 15.1 \\
\hline leucine-rich repeat & 17 & 16 & 16 & 2 & 12.5 \\
\hline DNA topoisomerase & 9 & 9 & 9 & 1 & 11.1 \\
\hline protein tyrosine & 10 & 10 & 9 & 1 & 11.1 \\
\hline cytochrome c & 20 & 18 & 13 & 1 & 7.6 \\
\hline protein transport & 16 & 16 & 14 & 1 & 7.1 \\
\hline elongation factor & 203 & 65 & 15 & 1 & 6.6 \\
\hline$A B C$ transporter & 33 & 33 & 33 & 2 & 6 \\
\hline ubiquitin-conjugating enzyme & 24 & 24 & 23 & 1 & 4.3 \\
\hline serine/threonine protein & 80 & 78 & 78 & 3 & 3.8 \\
\hline 605 ribosomal & 106 & 106 & 93 & 3 & 3.2 \\
\hline DNA repair & 37 & 36 & 35 & 1 & 2.8 \\
\hline RNA-binding protein & 80 & 72 & 71 & 2 & 2.8 \\
\hline hypothetical protein & 11812 & 10489 & 9735 & 122 & 1.2 \\
\hline protein kinase & 311 & 277 & 270 & 1 & 0.3 \\
\hline
\end{tabular}

1. \# genome occurrences = number of instances for the annotation in the genome.

2. \# array occurrences = number of annotated genes with probes on the arrays.

3. \# array candidates = number of annotated genes represented by probes on the arrays and which had probe density of 5 unique probes per 500 bp. Genes could have insufficient probe density due to repeat regions or they could have too few probes due to length of the gene (minimum of 5 probes).

4. \# sig CNV = CNV with a minimum log2 ratio difference of $+/-0.5$, for a minimum of 5 probes over a segment size of 500 bp in at least 5 test strains.

surprisingly, genes that are members of large families of surface proteins, such as the mucins, trans-sialidases, mucin-associated surface proteins (MASPs), and surface proteases are among those with the highest number of $\mathrm{CNV}$ among the strains tested, with type II mucins showing $\mathrm{CNV}$ over $70 \%$ of the time. Interestingly, protein kinases, a large and diverse family of related genes, as well as hypothetical proteins were the gene groups with the least association with hotspot regions, suggesting that all or nearly all of them may be under strict copy number control. Other genes that are relatively stable in terms of copy number included genes for ribosomal proteins and DNA repair, consistent with their expected essential roles. CNVs in gene groups represented by less than 5 genes are shown in Additional file 5.

The typing of T. cruzi into 6 discrete typing units (DTUs) is based upon a relatively small number of loci, including small subunit rRNA, elongation factor $1 \alpha$, actin, dihydrofolate reductase-thymidylate sythase, and trypanothione reductase among others $[11,12]$. To determine if this DTU classification system matched to patterns of CNV among typed strains, we 'typed' chromosomes based on their CNV patterns, creating CNV signature types (Additional file 4) and then grouped these CNV-typed T. cruzi strains by similarity in the number of shared chromosome types (Figure 3). The typing results suggest that there is substantially more genetic diversity among the type I strains than there is among the type II strains. This is consistent with recent microsatellite analyses of type I strain diversity [13]. However, the apparently greater diversity among type I versus type II strains observed in our study could be a result of the feature selection. Features for typing were selected by an unbiased computational 


\section{Chromosome}

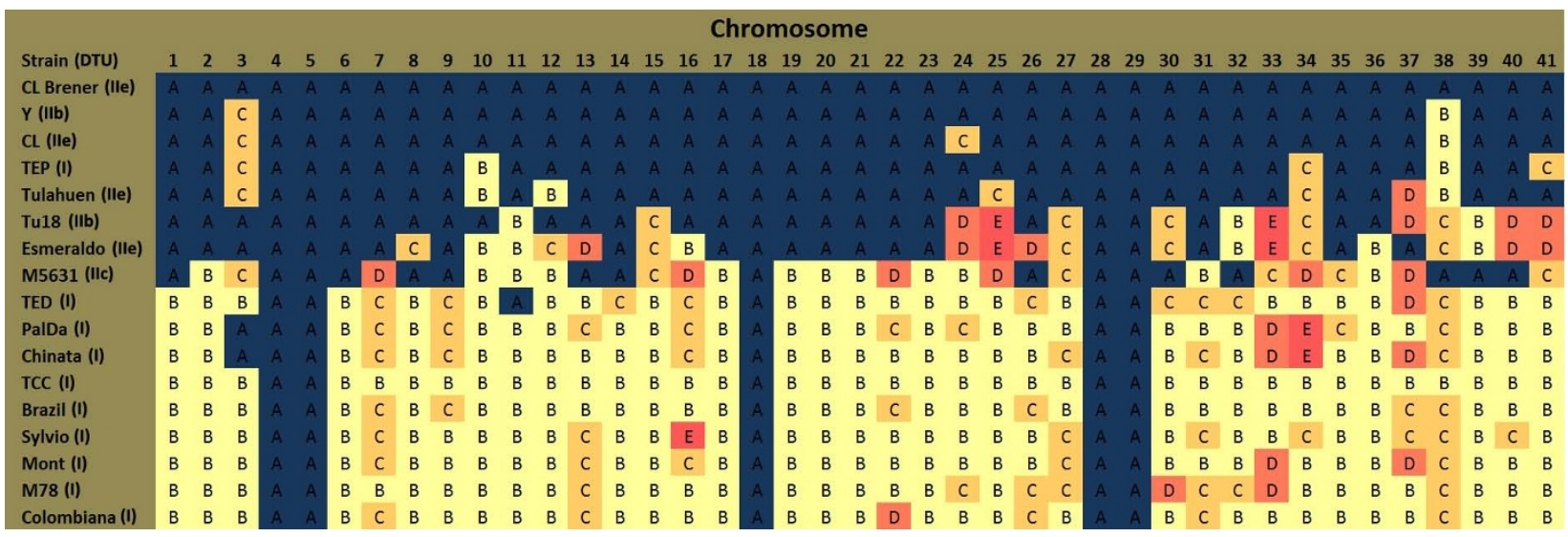

Figure 3 Chromosomes were typed based on CNV signatures. Diagnostic CNV were selected based on the criteria that they were at least $500 \mathrm{bp}$ in length, represented by at least 5 oligonucleotide probes, and had log2ratios of at least +/- 1 (tolerance of 0.2 ) in at least 5 strains. Up to two CNV meeting the criteria were selected for each chromosome based on their ability to differentiate between the strains; i.e. the CNV that separated the strains into the highest number of groups compared to other candidate diagnostic CNV were selected. Thus, for each

chromosome there were a finite number of signature types to which strains were assigned based upon the presence/absence of the diagnostic CNV's the direction of change (log2ratio +/- 0.8). Each chromosome type is represented by a different color, with CL-Brener always assigned to type ' $A$ ' (blue). The strains were then sorted based on the overall similarity of their chromosome types, taken together, relative to CL-Brener.

method, but not all of the nearly limitless possible combinations of available CNV features were used in the typing. Although type I and type II strains grouped together in the classification, on an individual chromosome basis some type I strains clearly had chromosomal $\mathrm{CNV}$ patterns matching those in type II strains and vice versa. For example, on chromosome 11 Esmeraldo, M5631, and Tu18 (types IIb, IIc, and IIb respectively) each have a CNV that is shared among all of the type I strains (Additional file 4, slide 11). Yet the other type II strains, including Y strain which is also type IIb, do not have this CNV. This CNV, a deletion, corresponds to the locus for proline racemase that may be a target of immune selection [14]. Taken together, these data suggest that either there is extensive homoplasy among $T$. cruzi strains (i.e. the same amplification/deletion events occurring multiple times in strains that are evolving independently) or that chromosome exchange has occurred more frequently in $T$. cruzi than the current two-hybridization event theory would suggest [5].

\section{Discussion}

In this study, we used whole-genome tiling arrays to catalog the diversity in gene copy number among $17 \mathrm{~T}$. cruzi strains with widely varying biological characteristics. The overwhelming conclusion from this work is that there is substantial and widespread variation in gene copy numbers among T. cruzi isolates. The observation of CNVs in T. cruzi is not surprising. The relative infrequency of sexual recombination, coupled with the near absence of transcriptional promoters in T. cruzi means that both the generation of genetic diversity and the regulation of the level of proteins is determined in part by within-isolate gene recombination and amplification. Nevertheless, the extent of variation in a relatively small set of isolates is quite dramatic. This variation is evident from the level of entire chromosomes down to individual genes, and CNVs tend to cluster in regions that are heavily populated with repeat sequences, including those encoding the members of gene families of the surface proteins, trans-sialidases, mucins, mucin associated surface proteins (MASP's), as well as retrotransposon hot spot (RHS) proteins, the dispersed gene family 1 (DGF-1), and protease gp63. Multicopy genes are frequently sources of mitotic recombination resulting in amplification and deletion of duplicate genes [15] so their relationship to regions of CNV is also not unexpected. The surface protein families in particular are known to be the major targets of protective immune responses and this selective pressure presumably drives their expansion and variation among strains.

In addition to the expected association of $\mathrm{CNV}$ with genes that are under immunological pressure, the presence and absence of CNV among other gene groups may be informative as to their functions and unique activities in T. cruzi. A relatively high fraction of serine carboxypeptidases, tryptophanyl aminoacyl tRNA synthetases, beta galactofuranosyl transferases and mitogen-activated protein kinases show CNVs among the $T$. cruzi isolates studied. The tryptophanyl aminoacyl tRNA synthetases are expanded to 10 distinct genes (not including pseudogenes) in T. cruzi whereas T. brucei and Leishmania each have only two, a cytoplasmic and a mitochondrial version. Interestingly, aminoacyl-tRNA 
synthetase genes in other eukaryotes have been reported to be increased in number and to acquire a diverse set non-enzymatic functions, including as inflammatory and angiogenic cytokines [16]. The location of this expanded set of the tryptophanyl tRNA sythetases genes in hotspots for recombination in $T$. cruzi also suggests that they may be modified and selected for as yet undetermined secondary functions. Similarly, T. cruzi has 37 beta-galactofuranosyl transferase genes.(compared to 2 for L. braziliensis, 1 each for L. major and L. infantum, and none for $T$. brucei) located in regions of high between-strain CNV. These enzymes are involved in the synthesis of glycans on mucins in T. cruzi. Mucin glycan structure is complex and heterogeneous between $T$. cruzi strains, again consistent with the expansion and variation in transferases among $T$. cruzi strains noted herein [17]. Conversely, only 1 of 270 protein kinases that passed the data filters for this analysis and $<2 \%$ of the nearly 10,000 hypothetical proteins were associated with hotspot regions, suggesting that variation, amplification and recombination of these genes is not tolerated or provides no selective advantage.

Although this analysis suggests extensive occurrence of whole chromosome aneuploidy in T. cruzi, the range of chromosome numbers as well as the absolute number of individual chromosomes in each strain cannot be estimated based upon our analysis. The decreased signal intensities evident across whole chromosomes in this $\mathrm{CGH}$ analysis would appear to be deletions but could also be in part due to sequence divergence in the test strains relative to the CL Brener reference sequences. Since individual isolates or strains of $T$. cruzi are thought to evolve without significant between-strain genetic exchange due to the relative absence of sexual recombination [1], the accumulation of sequence variants would be expected. Normally, traditional cytological methods such as metaphase spreads would be used to resolve karyotypic differences between isolates. However, this approach is not possible in the case of T. cruzi because $T$. cruzi replicates via endodyogeny, where the nuclear membrane does not break down and chromosomes do not fully condense during replication [18]. Nevertheless it seems highly unlikely that sequence divergence alone could explain our observations of chromosomes with significantly decreased signal intensities in many of the test strains relative to the CL-Brener reference. And certainly whole chromosome increases in signal relative to the reference strain cannot be accounted for by sequence divergence, since the target probes are based upon the reference strain sequence and diverged sequences are unlikely to out-compete the homologous sequences. For example, Y, Colombiana, M5631, wtCL, and TeDa2 each appear to have an extra chromosome 3 (Additional file 4, slide 3 ). It is most reasonable to interpret significant deviation from a log2 ratio of zero for test strain vs. reference strain over the length of a chromosome as being indicative of a real difference in the number of copies of that chromosome in the test and reference strains, supporting the conclusion that $T$. cruzi karyotypes are highly variable between strains and suggesting that there is perhaps no 'euploid' state for $T$. cruzi. This karyotypic plasticity may be another mechanism $T$. cruzi uses to generate diversity in spite of apparently being asexual or nearly so and may to some extent parallel what has been observed for the pathogenic yeast, Candida glabrata. Array CGH and pulsed field gel electrophoresis analyses of this haploid, asexual organism revealed extensive variability in the karyotypes of C. glabrata strains and suggest that this variability may be linked to drug resistance $[19,20]$.

The biological characteristics of $T$. cruzi strains have been used to classify isolates into biodemes [21,22] and as well the DTU classification has been associated to certain transmission and virulence characteristics of isolates [23-26]. Nevertheless, the link between DTU type and the biological characteristics of a strain are not strong [27]. Likewise, we find that the DTU organization of strains is a poor predictor of patterns of CNV in individual chromosomes. Thus, clear and specific patterns of amplifications and deletions in chromosomes are observed among strains, but these specific patterns are almost never restricted to or predictive of DTU type. This result has two important implications. First, the substantial and even continuous variation in biological characteristics of T. cruzi isolates will be difficult to account for using a limited number of genetic markers. Patterns of CNV provides another tool, but this too is not sufficient to predict behaviors that likely have a complex genetic basis. Nevertheless, these typing approaches do provide insights into the population structure of the species and the evolution of individual "strains" that are thought to be genetically isolated from each other. The results of typing of chromosomes by CNV patterns that we present herein provides new insights but also new questions related to genetic exchange in T. cruzi. The observation of shared patterns of CNV that do not track with the DTU type of a strain and are not consistent between chromosomes within the same strains can be explained in two ways: either such similar patterns of CNV are arising independently in isolates, perhaps due to common selective forces, or chromosomes of $T$. cruzi are being resorted or exchanged between isolates at a much higher frequency than is currently appreciated. The similarity and complexity of CNV would seem to favor the latter of these possibilities but a mechanism by which such exchange would occur is not clear. The full sequencing of additional T. cruzi isolates, as is currently underway, should 
help discriminate between these possibilities. Moreover, CGH arrays designed from the sequences of multiple $T$. cruzi strains, analogous to multi-species taxonomic arrays for Saccharomyces cerevisiae may help resolve the extent and nature of genetic exchange between T. cruzi strains and reveal heretofore undiscovered instances of introgression [28].

In addition to these insights into the biology and evolution of T. cruzi, the identification of "hot spots" for CNV within the $T$. cruzi genome should also provide guidance in the selection of candidates for vaccines and for targets for drug development. Although selecting candidates that are encoded outside these hot spots does not guarantee the absence of variation or the development of variation between isolates, avoiding genes that are in such hotspots would seem prudent.

\section{Conclusions}

CNV among T. cruzi strains are substantial in number, widespread throughout the $T$. cruzi genome, range in size from a few hundred base pairs to whole chromosomes (based upon the assembled CL-Brener genome), and are discordant with traditional DTU assignments for the strains tested. Taken together, these results suggest that there is much more genotypic diversity among T. cruzi strains than can be fathomed using traditional typing methods and that genetic exchange, possibly by the proposed "fusion then loss" mechanism [29], occurs between $T$. cruzi strains much more frequently than current dogma supposes. The large number of CNV shared between divergent DTU supports the genetic exchange hypothesis over the hypothesis that the shared $\mathrm{CNV}$ arose due to homoplasy.
CNV in $T$. cruzi tended to be concentrated in gene family rich regions of the genome, suggesting that the highly repetitive nature of the sequences in these regions is a strong driver of mitotic recombination in $T$. cruzi whether occurring within diploid strains or in strains resulting from hybridization events.

Neither the traditional DTU assignments for the $T$. cruzi strains used in this study nor the strain groupings based upon CNV patterns are currently good predictors of the biological characteristics of these strains. Thus, further $\mathrm{CGH}$ and sequencing studies of a larger panel of well-characterized T. cruzi strains is warranted to determine appropriate genotypic markers of T. cruzi biological diversity. Moreover, it would seem prudent to use the CNV data reported herein when selecting targets for drug and vaccine studies in order to avoid genes in hotspots of CNV occurrence.

\section{Methods}

\section{Parasites and DNA isolation}

$T$. cruzi strains were selected to represent a biologically diverse sample set from multiple DTU (Table 2). Epimastigotes were cultured in Liver Infusion Tryptose (LIT) medium and harvested as previously described [30]. Genomic DNA was obtained as previously described [31]. Briefly, epimastigotes were washed three times with ice-cold PBS, resuspended at a final density of $2 \mathrm{E}+08$ cells per $\mathrm{ml}$ in lysis buffer $(150 \mathrm{mM} \mathrm{NaCl}$, $100 \mathrm{mM}$ EDTA, $100 \mathrm{ug} / \mathrm{ml}$ proteinase $\mathrm{K}, 10 \mathrm{ug} / \mathrm{ml}$ RNase $\mathrm{A}$, and $0.5 \%$ sodium sarcosinate, $\mathrm{pH} 8.0$ ) and incubated at $50^{\circ} \mathrm{C}$ for $30 \mathrm{~m}$. Lysates were then extracted twice with phenol/chloroform and the DNA was ethanol precipitated, then resuspended in $5 \mathrm{mM}$ Tris. $\mathrm{Cl}, \mathrm{pH} 7.5$.

Table 2 Trypanosoma cruzi strains used in this study

\begin{tabular}{|c|c|c|c|c|c|c|}
\hline strain & origin & host & DTU & virulence & drug resistance & reference \\
\hline Brazil & Brazil & Human & 1 & high & low & {$[37]$} \\
\hline Chinata & Bolivia & Triatoma infestans & I & high & ND & unpublished \\
\hline Colombiana & Colombia & Human & I & high & moderate & {$[21]$} \\
\hline Esmeraldo & Brazil & Human & $\| \mathrm{lb}$ & moderate & ND & {$[38]$} \\
\hline M5631 & Brazil & Dasypus novemcinctus & $\| \mathrm{C}$ & ND & ND & {$[39]$} \\
\hline M78 & Argentina & Human & I & low & ND & {$[40]$} \\
\hline Montalvania & Brazil & Human & I & high & moderate & Andrade unpublished \\
\hline PalDa1 (clone 9) & Argentina & Didelphis albiventris & I & low & ND & {$[41]$} \\
\hline Sylvio X10/4 & Brazil & Human & 1 & low & ND & {$[42]$} \\
\hline TCC & unknown & unknown & I & low & ND & {$[43]$} \\
\hline TEDa2 (clone 4) & Argentina & Didelphis albiventris & I & ND & ND & {$[41]$} \\
\hline TEP6 (clone 5) & Argentina & dog & 1 & ND & ND & {$[41]$} \\
\hline Tu18 (clone 1) & Bolivia & Triatoma infestans & $\| b$ & ND & ND & {$[44]$} \\
\hline Tulahuen & Chile & Human & Ile & high & low & {$[45]$} \\
\hline wtCL & Brazil & Triatoma infestans & Ile & high & low & {$[46]$} \\
\hline Y & Brazil & Human & $\| \mathrm{b}$ & high & low & {$[47]$} \\
\hline CL-Brener & Brazil & Triatoma infestans & Ile & high & ND & {$[48]$} \\
\hline
\end{tabular}


DNA sample integrity was checked by agarose gel electrophoresis. Genomic DNA for CL-Brener strain and Esmeraldo strains were kindly provided by JM Kelly and B Zingales, respectively.

\section{Array Design}

Whole genome tiling arrays for comparative genome analysis were designed as previously described [32] with the notable exception that all the criteria related to sequence similarity with other regions of the genome had to be relaxed in $T$. cruzi in order to ensure proper coverage. We used 50-mer oligonucleotide probes and the microarray had a total capacity of approximately 380,000 probes, approximately $25 \%$ of which were used for preliminary quality assessment in this first generation design. The $\sim 290,000$ probes relevant for the current work were selected with the following procedure: 1 ) only the contigs including annotated genes were targeted, 2) the 50-mers occurring more than 4 times in the genome were eliminated, 3) the homopolymers longer than 5 nucleotides were eliminated, 4) only the 50-mer oligonucleotides with a melting temperature Tm within $+-5^{\circ} \mathrm{C}$ of the median melting temperature were kept (where $\mathrm{Tm}=0.41 \mathrm{GC}+$ constant), 5) the oligonucleotides requiring more than 150 cycles [33] to synthesize were eliminated, 6) the oligonucleotides with a selffolding energy smaller than $-1 \mathrm{kcal} / \mathrm{mol}$ according to a hybrid-ss-min calculation [34] were eliminated, and 7) from the probes passing all the above filters $\sim 290,000$ were selected with a strategy designed to maximize the uniformity of coverage of the entire genome. However, for some regions of the genome, gene family rich regions in particular, the DNA sequences were highly repetitive resulting in low tiling densities for those regions. For the same reason probes were not designed to sequencing scaffolds that were not used in the genome assembly. Construction of the arrays was performed by Roche NimbleGen Inc. (see reference [35] for additional details).

\section{Hybridization, Scanning, and Image Analysis}

Genomic DNA from T. cruzi strains was sonicated and labeled per the Nimblegen protocol. Labeling was by random-primed synthesis using $\mathrm{Cy} 3 / 5$-labeled random nonamers, and mixed probes were hybridized to the arrays per the manufacturer's protocol. Microarrays were scanned using a ScanArray 5000 (Perkin Elmer) and signal intensity files were generated using NimbleScan software. Ratios of fluorescence intensities were calculated without applying any background subtraction and the log2ratio values were normalized following a LOESS regression and visualized using the $\mathrm{R}$ statistical software [36]. The data from these experiments have been deposited in the Gene Expression Omnibus (http://www.ncbi.nlm.nih.gov/geo/) under the accession GSE23576.

\section{Additional material}

\begin{abstract}
Additional file 1: Microsoft PowerPoint file of theCGHViewer view of $T$. cruzi chromosome 35 showing the CNV generated by knockout of one copy each of ECH1 and ECH2 (enoyl-CoA hydratase/isomerase family protein; Tc00.1047053511529.160, Tc00.1047053511529.150). Each dot represents an oligonucleotide probe. The CL-Brener strain, which was used as the reference strain for genome sequencing, is hybrid, thus probes were designed to nonEsmeraldo (non-Esm) sequences (blue), Esmeraldo-like (Esm) sequences (green), non-Esm gene family sequences (black), and Esm gene family sequences (gray). Positive log2 ratios of signal intensities (wild type strain/knockout strain) represent deletion in the knockout strain and negative $\log 2$ ratios represent amplification in the knockout strain, relative to wt $T$. cruzi. Units for the $X$ axis (Position) are base pairs. Inset in panel $A$ is the GBrowse view of the locus ( $E C H$ genes purple circle). Panel $B$ is a close-up view of the locus on chromosome 35
\end{abstract}

Additional File 2: PowerPoint file of all of the array data ordered by chromosome. Representative plots from 16 hybridizations for each $T$. cruzi chromosome. Each dot represents an oligonucleotide probe. The $\mathrm{CL}$-Brener strain, which was used as the reference strain for genome sequencing, is hybrid, thus probes were designed to non-Esmeraldo (non-Esm) sequences (blue), Esmeraldo-like (Esm) sequences (green), non-Esm gene family sequences (black), and Esm gene family sequences (gray). In each panel positive log2 ratios of signal intensities (test strain/ reference) represent amplification in the test strain and negative log2 ratios represent deletion in the test strain, relative to $C L-B r e n e r$, which was the reference strain in all hybridizations. Boxed regions were the features selected for chromosome typing as explained in Figure 3. The different patterns observed for each chromosome are displayed at the bottom with letters corresponding to the typing letters in Figure 3. Blue boxes denote lower copy number in the test strain versus the reference strain, red boxes higher copy number in the test strain, and black boxes equal copy number in the test and reference strains. In each case for each chromosome, the CL-Brener type was the default type "A." Also, while two strains may have been assigned to the same CNV signature type for a given chromosome, they were not necessarily identical for that chromosome, as not every single CNV for every single chromosome was used in the typing (such an analysis would render every chromosome for every strain unique and make finding common patterns impossible). Note that chromosomes 4, 5, 18, 28, and 29 did not present sufficiently informative typing regions. Thus, all strains were type " $A$ " for these chromosomes. Also, the CNV were haplotype specific. Therefore, in some cases this made the up or down calls (box color) appear incorrect, especially if the log2ratio for the feature was off the scale making it appear as if the boxed region is referring to the other haplotype. For example see PalDa for chromosome 8. In the boxed region the green (Esm) probes appear to be up yet the box is blue, indicating lower copy number, because the feature refers to non-Esm probes which are off the scale of the figure. Lastly, the boxes are guides to identifying the CNV used for typing, but they do not represent the exact bounds of those typing regions. In some cases due to the scale of the figure CNV that are close to the typing CNV appear to be part of the typing CNV.

Additional file 3: A java-based executable file for viewing all of the CGH array data from this study. The CGH data were visualized and explored using 'CGH_Viewer,' which was written in the Java programming language. Additional file 2 is a Windows executable (.exe) that will install the CGH_Viewer, along with Java if it is not detected, on the target computer. The CGH_Viewer takes as input the mapping of probes from the microarray to the assembled chromosomes of $T$. cruzi as well as the results of 1 or more experiments between 2 strains. The probe-to-chromosome mapping file and the 16 experimental files are provided in the "Data" sub-folder. The results (dot plots of log2 ratios) of multiple chromosomes and multiple experiments may be viewed simultaneously. Zooming on an area of interest on a chromosome will show the same region across all experiments. The data can be filtered 
based on 1) the haplotype of sequence from which the probes were designed as well, 2) the ID or name of the sequence from which the probe was designed, 3 ) by raw intensity of the probe (a quality measurement), or 4) a sub-region of a chromosome. For additional information, see the provided documentation in the CGH_Viewer program folder (c:IProgram Files\CGH_Viewer))

Additional file 4: Excel file containing the average normalized log2 ratios of signal intensities (test strain/CL Brener) for each coding and non-coding region in the $T$. cruzi genome for each of the hybridizations performed in this study. Esmeraldo-like (Esm) and nonEsmeraldo (non-Esm) probes for the indicated regions are averaged separately. Density is the genomic range (in base pairs) divided by the number of probes covering that range.

Additional file 5: MSWord file containing a chart of the distribution of genes associated with hotspot regions having less than 5 candidates on the arrays. Candidates were determined by having at least 5 probes within a maximum sequence size of $500 \mathrm{bp}$.

\section{Acknowledgements}

We acknowledge Angel Padillia, Patricio Diosque, Miriam Postan, and Robert Sabatini for supplying T. cruzi strains, and John Kelly and Bianca Zingales for supplying CL-Brener and Esmeraldo DNA, respectively. We also acknowledge Charles Rosenberg for helpful discussions. Lastly, we acknowledge Dan Xu for providing DNA for the initial validation experiments. RLT, TM, and DBW were supported by for this work by P01Al044979.

\section{Author details}

'Center for Tropical and Emerging Global Diseases, University of Georgia, Athens, Georgia, 30602, USA. '2Department of Zoology, University of British Columbia, Vancouver, British Columbia V6T 1Z4, Canada.

\section{Authors' contributions}

TAM designed and performed the microarray experiments, analyzed and interpreted the data, and wrote the manuscript. DBW assisted in the bioinformatics and data analysis and wrote the CGH_Viewer program. SF designed the microarrays, performed validation experiments, and wrote the within-hybridization normalization program. RLT initiated and guided the project, analyzed and interpreted data, and wrote the manuscript. All authors have read and approved the final manuscript.

Received: 31 August 2010 Accepted: 7 March 2011 Published: 7 March 2011

\section{References}

1. Tibayrenc M, Ward P, Moya A, Ayala FJ: Natural populations of Trypanosoma cruzi, the agent of Chagas disease, have a complex multiclonal structure. Proc Natl Acad Sci USA 1986, 83(1):115-119.

2. Woo PT, Soltys MA: Animals as reservoir hosts of human trypanosomes. Journal of wildlife diseases 1970, 6(4):313-322

3. Tibayrenc M: Genetic subdivisions within Trypanosoma cruzi (Discrete Typing Units) and their relevance for molecular epidemiology and experimental evolution. Kinetoplastid Biol Dis 2003, 2(1):12

4. Zingales B, Andrade SG, Briones MR, Campbell DA, Chiari E, Fernandes O, Guhl F, Lages-Silva E, Macedo AM, Machado CR, Miles MA, Romanha AJ, Sturm NR, Tibayrenc M, Schijman AG: A new consensus for Trypanosoma cruzi intraspecific nomenclature: second revision meeting recommends Tcl to TcVI. Mem Inst Oswaldo Cruz 2009, 104(7):1051-1054

5. Westenberger SJ, Barnabe C, Campbell DA, Sturm NR: Two hybridization events define the population structure of Trypanosoma cruzi. Genetics 2005, 171(2):527-543.

6. El-Sayed NM, Myler PJ, Bartholomeu DC, Nilsson D, Aggarwal G, Tran AN, Ghedin E, Worthey EA, Delcher AL, Blandin G, et al: The genome sequence of Trypanosoma cruzi, etiologic agent of Chagas disease. Science 2005, 309(5733):409-415

7. Martin DL, Weatherly DB, Laucella SA, Cabinian MA, Crim MT, Sullivan S, Heiges M, Craven SH, Rosenberg CS, Collins MH, Sette A, Postan M, Tarleton RL: CD8+ T-Cell responses to Trypanosoma cruzi are highly focused on strain-variant trans-sialidase epitopes. PLoS pathogens 2006, 2(8):e77.

8. Buscaglia CA, Campo VA, Frasch AC, Di Noia JM: Trypanosoma cruzi surface mucins: host-dependent coat diversity. Nature reviews 2006, 4(3):229-236.

9. Weatherly DB, Boehlke C, Tarleton RL: Chromosome level assembly of the hybrid Trypanosoma cruzi genome. BMC genomics 2009, 10:255.

10. Xu D, Brandan CP, Basombrio MA, Tarleton RL: Evaluation of high efficiency gene knockout strategies for Trypanosoma cruzi. BMC microbiology 2009, 9:90

11. Brisse S, Dujardin JC, Tibayrenc M: Identification of six Trypanosoma cruzi lineages by sequence-characterised amplified region markers. Mol Biochem Parasitol 2000, 111(1):95-105.

12. Tomazi L, Kawashita SY, Pereira PM, Zingales B, Briones MR: Haplotype distribution of five nuclear genes based on network genealogies and Bayesian inference indicates that Trypanosoma cruzi hybrid strains are polyphyletic. Genet Mol Res 2009, 8(2):458-476.

13. Llewellyn MS, Miles MA, Carrasco HJ, Lewis MD, Yeo M, Vargas J, Torrico F, Diosque P, Valente V, Valente SA, Gaunt MW: Genome-scale multilocus microsatellite typing of Trypanosoma cruzi discrete typing unit I reveals phylogeographic structure and specific genotypes linked to human infection. PLoS pathogens 2009, 5(5):e1000410.

14. Reina-San-Martin B, Degrave W, Rougeot C, Cosson A, Chamond N, Cordeiro-Da-Silva A, Arala-Chaves M, Coutinho A, Minoprio P: A B-cell mitogen from a pathogenic trypanosome is a eukaryotic proline racemase. Nature medicine 2000, 6(8):890-897.

15. Hurles M: Gene duplication: the genomic trade in spare parts. PLOS biology 2004, 2(7):E206.

16. Brown MV, Reader JS, Tzima E: Mammalian aminoacyl-tRNA synthetases: cell signaling functions of the protein translation machinery. Vascular pharmacology 52(1-2):21-26.

17. Jones C, Todeschini AR, Agrellos OA, Previato JO, Mendonca-Previato L: Heterogeneity in the biosynthesis of mucin O-glycans from Trypanosoma cruzi tulahuen strain with the expression of novel galactofuranosyl-containing oligosaccharides. Biochemistry 2004, 43(37):11889-11897.

18. Spadiliero B, Nicolini C, Mascetti G, Henriquez D, Vergani L: Chromatin of Trypanosoma cruzi: In situ analysis revealed its unusual structure and nuclear organization. Journal of cellular biochemistry 2002, 85(4):798-808.

19. Polakova S, Blume C, Zarate JA, Mentel M, Jorck-Ramberg D, Stenderup J, Piskur J: Formation of new chromosomes as a virulence mechanism in yeast Candida glabrata. Proc Natl Acad Sci USA 2009, 106(8):2688-2693.

20. Muller H, Thierry A, Coppee JY, Gouyette C, Hennequin C, Sismeiro O, Talla E, Dujon B, Fairhead C: Genomic polymorphism in the population of Candida glabrata: gene copy-number variation and chromosomal translocations. Fungal Genet Biol 2009, 46(3):264-276.

21. Andrade S: Caracterizacao de cepas do Trypanosoma cruzi isoladas no Reconcavo Bahiano. Revista de Patologica Tropical 1974, 3:65-121.

22. Andrade SG, Magalhaes JB: Biodemes and zymodemes of Trypanosoma cruzi strains: correlations with clinical data and experimental pathology. Rev Soc Bras Med Trop 1996, 30(1):27-35.

23. Zingales B, Stolf BS, Souto RP, Fernandes O, Briones MR: Epidemiology, biochemistry and evolution of Trypanosoma cruzi lineages based on ribosomal RNA sequences. Mem Inst Oswaldo Cruz 1999, 94(Suppl 1):159-164

24. Briones MR, Souto RP, Stolf BS, Zingales B: The evolution of two Trypanosoma cruzi subgroups inferred from rRNA genes can be correlated with the interchange of American mammalian faunas in the Cenozoic and has implications to pathogenicity and host specificity. Mol Biochem Parasitol 1999, 104(2):219-232.

25. Hall CA, Polizzi C, Yabsley MJ, Norton TM: Trypanosoma cruzi prevalence and epidemiologic trends in lemurs on St. Catherines Island, Georgia. $J$ Parasitol 2007, 93(1):93-96.

26. Llewellyn MS, Lewis MD, Acosta N, Yeo M, Carrasco HJ, Segovia M, Vargas J, Torrico F, Miles MA, Gaunt MW: Trypanosoma cruzi Ilc: phylogenetic and phylogeographic insights from sequence and microsatellite analysis and potential impact on emergent Chagas disease. PLoS neglected tropical diseases 2009, 3(9):e510.

27. del Puerto R, Nishizawa JE, Kikuchi M, lihoshi N, Roca Y, Avilas C, Gianella A, Lora J, Velarde FU, Renjel LA, Miura S, Higo H, Komiya N, Maemura K, Hirayama K: Lineage analysis of circulating Trypanosoma cruzi parasites 
and their association with clinical forms of Chagas disease in Bolivia. PLoS neglected tropical diseases 4(5):e687.

28. Muller LA, McCusker JH: A multispecies-based taxonomic microarray reveals interspecies hybridization and introgression in Saccharomyces cerevisiae. FEMS yeast research 2009, 9(1):143-152.

29. Gaunt MW, Yeo M, Frame IA, Stothard JR, Carrasco HJ, Taylor MC, Mena SS, Veazey P, Miles GA, Acosta N, de Arias AR, Miles MA: Mechanism of genetic exchange in American trypanosomes. Nature 2003, 421(6926):936-939.

30. Minning TA, Weatherly DB, Atwood J, Orlando R, Tarleton RL: The steadystate transcriptome of the four major life-cycle stages of Trypanosoma cruzi. BMC genomics 2009, 10:370

31. Ozaki LS, Cseko YMT: Genomic cloning and related techniques. In Genes and antigens of parasites: A laboratory manual. second edition. Edited by: Morel CM. Rio de Janeiro, Brazil: Dept. of Biochemistry and Molecular Biology, Instituto Oswaldo Cruz; 1984.

32. Flibotte S, Moerman DG: Experimental analysis of oligonucleotide microarray design criteria to detect deletions by comparative genomic hybridization. BMC genomics 2008, 9:497.

33. Singh-Gasson S, Green RD, Yue Y, Nelson C, Blattner F, Sussman MR, Cerrina F: Maskless fabrication of light-directed oligonucleotide microarrays using a digital micromirror array. Nature biotechnology 1999 17(10):974-978.

34. Markham N: Hybrid: A software system for nucleic acid folding, hybridizing, and melting conditions. Rensselaer Polytechnic Institute Troy, NY; 2003.

35. Maydan JS, Flibotte S, Edgley ML, Lau J, Selzer RR, Richmond TA, Pofahl NJ, Thomas JH, Moerman DG: Efficient high-resolution deletion discovery in Caenorhabditis elegans by array comparative genomic hybridization. Genome research 2007, 17(3):337-347.

36. R: A language and environment for statistical computing. [http://www.Rproject.org].

37. Pan SC: Establishment of clones of Trypanosoma cruzi and their characterization in vitro and in vivo. Bulletin of the World Health Organization 1982, 60(1):101-107.

38. Miles MA, Toye PJ, Oswald SC, Godfrey DG: The identification by isoenzyme patterns of two distinct strain-groups of Trypanosoma cruzi, circulating independently in a rural area of Brazil. Trans $R$ Soc Trop Med Hyg 1977, 71(3):217-225.

39. Miles MA, Povoa MM, de Souza AA, Lainson R, Shaw JJ, Ketteridge DS: Chagas's disease in the Amazon Basin: li. The distribution of Trypanosoma cruzi zymodemes 1 and 3 in Para State, north Brazil. Trans R Soc Trop Med Hyg 1981, 75(5):667-674

40. Engel JC, Dvorak JA, Segura EL, Crane MS: Trypanosoma cruzi: biological characterization of 19 clones derived from two chronic chagasic patients. I. Growth kinetics in liquid medium. The Journal of protozoology 1982, 29(4):555-560.

41. Diosque P, Barnabe C, Padilla AM, Marco JD, Cardozo RM, Cimino RO, Nasser JR, Tibayrenc M, Basombrio MA: Multilocus enzyme electrophoresis analysis of Trypanosoma cruzi isolates from a geographically restricted endemic area for Chagas' disease in Argentina. Int J Parasitol 2003, 33(10):997-1003.

42. Postan M, Dvorak JA, McDaniel JP: Studies of Trypanosoma cruzi clones in inbred mice. I. A comparison of the course of infection of $\mathrm{C} 3 \mathrm{H} / \mathrm{HEN}$ mice with two clones isolated from a common source. Am J Trop Med Hyg 1983, 32(3):497-506.

43. Basombrio MA, Besuschio S, Cossio PM: Side effects of immunization with liver attenuated Trypanosoma cruzi in mice and rabbits. Infect Immun 1982, 36(1):342-350.

44. Breniere SF, Bosseno MF, Telleria J, Bastrenta B, Yacsik N, Noireau F, Alcazar JL, Barnabe C, Wincker P, Tibayrenc M: Different behavior of two Trypanosoma cruzi major clones: transmission and circulation in young Bolivian patients. Exp Parasitol 1998, 89(3):285-295.

45. Cariola J, Prado R, Agosin M, Christen R: Susceptibility of the hamster and Peromyscus to experimental Trypanosoma cruzi infection (Tulahuen strain). Boletin de informaciones parasitarias chilenas 1950, 5(4):44-45

46. Brener Z, Chiari E: Morphological Variations Observed in Different Strains of Trypanosoma Cruzi. Rev Inst Med Trop Sao Paulo 1963, 19:220-224.

47. Carvalheiro Jda R, Collares EF: Studies on the behavior, in mice, of a highly virulent strain (Y strain) of Trypanosoma cruzi after passage in
Triatoma, rats and cultures. Revista brasileira de biologia 1965, 25(2):169-175.

48. Brisse S, Barnabe C, Banuls AL, Sidibe I, Noel S, Tibayrenc M: A phylogenetic analysis of the Trypanosoma cruzi genome project $\mathrm{CL}$ Brener reference strain by multilocus enzyme electrophoresis and multiprimer random amplified polymorphic DNA fingerprinting. Mol Biochem Parasitol 1998, 92(2):253-263.

doi:10.1186/1471-2164-12-139

Cite this article as: Minning et al:: Widespread, focal copy number variations (CNV) and whole chromosome aneuploidies in Trypanosoma cruzi strains revealed by array comparative genomic hybridization. BMC Genomics 2011 12:139.

\section{Submit your next manuscript to BioMed Central and take full advantage of:}

- Convenient online submission

- Thorough peer review

- No space constraints or color figure charges

- Immediate publication on acceptance

- Inclusion in PubMed, CAS, Scopus and Google Scholar

- Research which is freely available for redistribution

Submit your manuscript at www.biomedcentral.com/submit
C Biomed Central 